DOI: 10.12957/demetra.2018.29331

\title{
Frequência de aparecimento de critérios sugestivos para transtorno da compulsão alimentar periódica em pacientes atendidos no ambulatório de nutrição da Faculdade de Nutrição Emília de Jesus Ferreiro, da Universidade Federal Fluminense
}

\section{Frequency of appearance of suggestive criteria for periodic food completion disorder in patients served in the nutrition ambulatory of Emília de Jesus Ferreiro Nutrition School, Universidade Federal Fluminense}

Charlene de Abreu Silva'

Kelly Beraldo Cardoso Grassano'

Sérgio Girão Barroso?

Gabrielle de Souza Rocha

${ }^{1}$ Universidade Federal Fluminense, Faculdade de Nutrição Emília de Jesus Ferreiro. Niterói, RJ, Brasil.

${ }^{2}$ Universidade Federal Fluminense, Departamento de Nutrição e Dietética, Faculdade de Nutrição Emília de Jesus Ferreiro, Programa de Pós-graduação em Ciências da Nutrição. Niterói, RJ, Brasil.

Correspondência / Correspondence

Charlene de Abreu Silva

E-mail: leny_309@hotmail.com

\section{Resumo}

Estudo transversal objetivou identificar a frequência de aparecimento de critérios sugestivos para transtorno da compulsão alimentar periódica (TCAP), analisar a observância à dieta e determinar o estado nutricional dos pacientes atendidos no Ambulatório de Nutrição da Faculdade de Nutrição Emília de Jesus Ferreiro da Universidade Federal Fluminense. Participaram do estudo 30 indivíduos, ambos os sexos, com idades entre 18 e 60 anos, com índice de massa corporal entre $20,33 \mathrm{~kg} / \mathrm{m}^{2}$ e $49,21 \mathrm{~kg} / \mathrm{m}^{2}$. Para análise da presença de critérios sugestivos de TCAP, foi utilizada a Escala de Compulsão Alimentar Periódica (Binge Eating Scale). Para análise de observância à dieta, foi observada a perda de peso durante as consultas e adesão ao tratamento através da verificação dos prontuários. A avaliação antropométrica incluiu peso corporal, estatura, índice de massa corporal, circunferência abdominal, circunferência de pescoço e índice de conicidade. A amostra foi composta por 20 mulheres e 10 homens, e de acordo com o questionário, $63 \%(\mathrm{n}=19)$ pacientes não apresentaram critérios sugestivos para TCAP, enquanto $37 \%$ $(\mathrm{n}=11)$ apresentaram. Com relação ao aparecimento de critérios sugestivos para TCAP, o mesmo estava associado com a observância à dieta $(\mathrm{p}<0,05)$ e ao índice de massa corporal $(\mathrm{p}<0,05)$, porém não 
foram encontradas associações estatisticamente significativas com relação a circunferência abdominal, circunferência de pescoço e índice de conicidade. A presença de critérios sugestivos para TCAP está associada à não observância à dieta e a elevados valores de índice de massa corporal.

Palavras-chave: Transtorno da Compulsão Alimentar Periódica. Avaliação Nutricional. Nutrição.

\section{Abstract}

This study aimed to identify suggestive criteria for Binge Eating Disorder (BED) frequency, analyze dietary compliance, and to determine nutritional status of patients attended at the Nutrition Clinic at Emília de Jesus Ferreiro Nutrition School, Federal Fluminense University. Thirty individuals of both genders, aged between 18 and 60 years, with body mass index between 20.33 $\mathrm{kg} / \mathrm{m} 2$ and $49.21 \mathrm{~kg} / \mathrm{m} 2$ participated in the study. To analyze presence of suggestive criteria for eating compulsion, the Binge Eating Scale was used. For dietary compliance analysis, weight loss was observed during consultations and compliance through the clinical records. Anthropometric evaluation included body weight, height, abdominal circumference, neck circumference and conicity index. Sample included 20 women and 10 men, and according to the questionnaire, $63 \%(\mathrm{n}=19)$ of the out patients presented no suggestive criteria, while $37 \%(\mathrm{n}=11)$ presented suggestive criteria. Regarding suggestive criteria for BED it was associated with dietary compliance $(p<0.05)$ and body mass index ( $\mathrm{p}<0.05)$, but not with abdominal circumference, neck circumference and conicity index. In conclusion, suggestive criteria for BED is associated with diet non-compliance and high values of body mass index.

Keywords: Binge Eating Disorder. Nutritional Assessment. Nutrition. 


\section{Introdução}

O sedentarismo e a obesidade são duas condições consideradas de risco ao indivíduo e à sociedade. ${ }^{1}$ Diferentes pesquisas têm demonstrado que ambas apresentam elevadas taxas de prevalência no mundo e no Brasil, constituindo verdadeiras epidemias. ${ }^{2,3}$

Têm-se observado significativas modificações no padrão alimentar da população em muitos países, inclusive no Brasil, onde se destacam o consumo excessivo de açúcares, gorduras, produtos industrializados, ricos em sódio, além da redução da ingestão do feijão com arroz e a insuficiência no consumo de frutas e hortaliças. ${ }^{4-6}$

A abordagem inicial do problema seria o tratamento clínico nutricional, estímulo à prática de atividades físicas, associando-se a terapia comportamental. Em pacientes com obesidade graus II e III, contudo, a combinação dos tratamentos comportamental e farmacológico tem tido pouco sucesso e, muitas vezes, é acompanhada de reganho de peso. Esgotadas as chances de sucesso do tratamento clínico, indica-se o tratamento cirúrgico. ${ }^{7}$

Sendo assim, atingir o peso corporal ideal é certamente desafiador no ambiente obesogênico em que vivemos. Outros fatores ambientais tendem a influenciar o ganho de peso gradual na população e em obesos causam dificuldades na adesão ao tratamento, bem como o aparecimento de transtornos alimentares. ${ }^{8}$

A obesidade não está inclusa no Manual Diagnóstico e Estatístico de Transtornos Mentais (DSM V - 2013); ${ }^{9}$ existem, no entanto, associações robustas entre a obesidade e uma série de transtornos mentais, entre eles: transtornos alimentares, transtornos depressivo e bipolar e esquizofrenia. Dentre os transtornos alimentares, a obesidade está associada à bulimia nervosa (BN) e ao transtorno da compulsão alimentar periódica (TCAP). ${ }^{10}$

O transtorno da compulsão alimentar periódica (TCAP) corresponde a episódios de compulsão alimentar juntamente com a perda de controle. Para ser classificado como TCAP, devem estar presentes pelo menos três dos seguintes comportamentos: comer rapidamente, comer até sentirse cheio, comer grandes quantidades de comida mesmo sem estar com fome, comer sozinho por embaraço pela quantidade de comida, sentir repulsa por si mesmo, depressão ou demasiada culpa após compulsão. Além disso, uma acentuada angústia pela compulsão alimentar, a frequência e duração da compulsão alimentar devem ocorrer, em média, ao menos uma vez por semana durante três meses. ${ }^{11}$

Ainda que o TCAP não se encontre limitado a indivíduos obesos, é um diagnóstico frequentemente observado neste grupo, especialmente naqueles que procuram tratamento para perda peso. Enquanto a prevalência estimada de TCAP na população geral pode variar de 1,5 a $5 \%$, em amostras clínicas de pacientes obesos encontra-se em torno de 7,5 a $30 \% .^{10}$ 
Devido à má alimentação, dificuldades de adesão ao tratamento nutricional, sedentarismo e baixa participação em programas de exercício físico, indivíduos obesos têm demonstrado maior suscetibilidade ao aparecimento de TCAP. ${ }^{12}$ Sendo assim, torna-se necessário investigar a presença de critérios sugestivos para TCAP nesses pacientes.

O objetivo deste estudo foi identificar a frequência de aparecimento de critérios sugestivos para TCAP, analisar a observância à dieta e determinar o estado nutricional dos pacientes atendidos no Ambulatório de Nutrição da Faculdade de Nutrição Emília de Jesus Ferreiro, da Universidade Federal Fluminense (UFF).

\section{Métodos}

Trata-se de estudo transversal em que informações pessoais foram coletadas diretamente do prontuário do paciente, sendo os dados antropométricos aferidos no momento da consulta. Participaram todos os pacientes, de ambos os sexos, com idade entre 18 e 59 anos e que foram atendidos no Ambulatório de Nutrição da Faculdade de Nutrição Emília de Jesus Ferreiro da UFF.

Foram considerados como critérios de exclusão: gestantes, idosos, presença de massas cervicais ou deformidades no pescoço, bócio, pacientes com doença crônica descompensada, doença renal crônica ou câncer.

O estudo foi aprovado pelo Comitê de Ética e Pesquisa da Universidade Federal Fluminense sob o número de registro CAAE 59613416.6.0000.5243. Cada etapa da pesquisa foi lida e explicada para o paciente e, mediante a aceitação em participar de forma voluntária do estudo, o paciente assinou o Termo de Consentimento Livre Esclarecido (TCLE).

Para análise da presença de comportamento compulsivo na ingestão alimentar, foi utilizada a Escala de Compulsão Alimentar Periódica (Binge Eating Scale - BES). A BES é um questionário autoaplicável, válido como instrumento de rastreamento da compulsão alimentar periódica (CAP). ${ }^{13}$ O questionário possui 16 questões, contendo 62 afirmativas, das quais deve ser selecionada, em cada questão, aquela que melhor representa a resposta do indivíduo. Cada afirmativa possui um número de pontos de 0 a 3, abrangendo desde a ausência ("0”) até a gravidade máxima (“3”) da compulsão alimentar periódica. O escore final é o resultado da soma dos pontos de cada questão. Indivíduos com pontuação menor ou igual a 17 são considerados sem compulsão; com pontuação entre 18 e 26 são considerados com compulsão moderada; e aqueles com pontuação maior ou igual a 27 , com a forma grave. ${ }^{14}$

Com relação à observância a dieta, consideramos como observantes os pacientes que apresentaram perda de peso e compareceram assiduamente às consultas, e não observantes os pacientes que não apresentaram perda de peso e não compareceram assiduamente às consultas, sendo mantidos no 
presente trabalho pacientes que não apresentaram adesão ao tratamento nutricional proposto, para análise da associação entre presença de critérios para TCAP e não observância à dieta.

A classificação do estado nutricional foi baseada no cálculo do índice de massa corporal (IMC) segundo a Organização Mundial da Saúde (OMS), ${ }^{15}$ sendo: eutrofia (IMC = 18,5-24,9kg/m²); sobrepeso $\left(\mathrm{IMC}=25,0-29,9 \mathrm{~kg} / \mathrm{m}^{2}\right)$; e obesidade $\left(\mathrm{IMC} \geq 30,0 \mathrm{~kg} / \mathrm{m}^{2}\right)$. A circunferência abdominal (CA) foi avaliada de acordo com o National Cholesterol Education Program (NCEP) - Adult Treatment Panel III (ATPIII) em 2000, ${ }^{16}$ sendo utilizados os pontos de corte de $102 \mathrm{~cm}$ para homens e 88 $\mathrm{cm}$ para mulheres.

Foram utilizados esses pontos de referências visto que, ao utilizar os pontos de corte da American Heart Associantion (AHA) em 2009, ${ }^{17}$ praticamente todos os pacientes estariam classificados como acima do ponto de corte, o que dificultaria a análise estatística.

A circunferência de pescoço (CP) foi utilizada como um indicador antropométrico, sendo classificada como elevada maior ou igual a $37 \mathrm{~cm}$ para homens e maior ou igual a $34 \mathrm{~cm}$ para mulheres, conforme estudo de Ben-Noun et al. ${ }^{18}$

O índice de conicidade (índice $\mathrm{C}$ ) foi calculado através da fórmula de Valdez, ${ }^{19} \mathrm{e}$ através do cálculo da mediana considerou-se 1,28 como melhor ponto de corte para homens, e 1,32 para mulheres, visto que praticamente todos os pacientes estariam classificados como acima do ponto de corte sugerido na literatura, dificultando a análise estatística.

Para determinação da análise do estudo, foi utilizado o Teste Exato de Fischer, para calcular as razões de chance $(\mathrm{RC})$ da presença ou não de critérios sugestivos para TCAP, observância à dieta e medidas antropométricas. Foram considerados significativos os resultados quando $\mathrm{p}<$ 0,05. O programa utilizado foi o Graph Pad Prism 5.0.

\section{Resultados}

Participaram do estudo 30 pacientes atendidos no Ambulatório de Nutrição da Faculdade de Nutrição Emília de Jesus Ferreiro, da Universidade Federal Fluminense, sendo 33\% (n=10) do sexo masculino e $67 \%(n=20)$ do sexo feminino. A amostra foi composta por indivíduos com idades entre 18 e 59 anos.

Com relação à escolaridade, apresentaram níveis desde o ensino fundamental até pósgraduação. A tabela 1 apresenta a caracterização geral da população analisada.

De acordo com o questionário ECAP, $63 \%(\mathrm{n}=19)$ dos pacientes não apresentaram critérios sugestivos para TCAP, enquanto 37\% $(n=11)$ dos pacientes apresentaram critérios sugestivos. Os resultados dos escores do ECAP são apresentados na tabela 2. 
Tabela 1. Caracterização geral da amostra. Niterói-RJ, 2017.

\begin{tabular}{l|c|c}
\hline \multicolumn{1}{c|}{ Idade } & Média \pm DP & Min - Máx \\
\hline Idade (anos) & $38,96 \pm 8,48$ & $18-59$ \\
\hline Escolaridade & $\mathrm{N}$ & $\%$ \\
\hline Ensino Fundamental & 3 & 10 \\
Ensino Médio & 13 & 44 \\
Ensino Superior & 10 & 33 \\
Pós-Graduação & 4 & 13 \\
\hline
\end{tabular}

Tabela 2. Resultados da Escala de Compulsão Alimentar periódica da amostra. Niterói RJ, 2017.

\begin{tabular}{l|c|c|c|c|c|}
\hline & N & $\%$ & Média \pm DP & Mín - Máx & Valor de Referência \\
\hline Sem CA & 19 & 63 & $7,05 \pm 5,60$ & $0-17$ & $<17$ \\
CA moderada & 7 & 24 & $22 \pm 4,24$ & $19-26$ & $18-26$ \\
CA grave & 4 & 13 & $31,5 \pm 2,12$ & $28-33$ & $>27$ \\
\hline
\end{tabular}

Legenda: Sem CA - Sem Compulsão Alimentar; CA moderada - Compulsão Alimentar moderada; CA grave Compulsão Alimentar grave.

De acordo com o sexo, $30 \%(\mathrm{n}=3)$ dos homens apresentaram critérios sugestivos para TCAP, enquanto $40 \%(n=8)$ das mulheres apresentaram critérios sugestivos para TCAP, conforme mostra a tabela 3 .

A tabela 4 apresenta os dados relativos à caracterização antropométrica da amostra. De acordo com o IMC, $10 \%(n=3)$ dos pacientes apresentaram estado nutricional de sobrepeso e $53 \%(n=16)$ eram obesos.

A tabela 5 apresenta os dados relativos à associação entre a presença ou não de critérios sugestivos para TCAP e observância a dieta, bem como a presença ou não de critérios sugestivos para TCAP e IMC. Destacam-se os resultados relativos a pacientes não observantes à dieta que estão associados à presença de critérios sugestivos de TCAP. O mesmo acontece com pacientes que apresentaram maiores índices de massa corporal; os demais parâmetros não apresentaram resultados estatisticamente significativos. 
Tabela 3. Resultados da Escala de Compulsão Alimentar Periódica por sexo. Niterói-RJ, 2017.

\begin{tabular}{l|c|c|c|c|c}
\hline \multicolumn{1}{c|}{ Homens } & $\mathrm{N}$ & $\%$ & Média \pm DP & Min-Máx & Valores de Referência \\
\hline Sem CA & 7 & 70 & $6,28 \pm 5,85$ & $0-16$ & $<17$ \\
CA moderada & 3 & 30 & $24 \pm 1,41$ & $23-26$ & $18-26$ \\
CA grave & 0 & - & - & - & $>27$ \\
\hline Mulheres & $\mathrm{N}$ & $\%$ & Média \pm DP & Min-Máx & Valores de Referência \\
\hline Sem CA & 12 & 60 & $7,5 \pm 5,66$ & $0-17$ & $<17$ \\
CA moderada & 4 & 20 & $22 \pm 4,24$ & $19-26$ & $18-26$ \\
CA grave & 4 & 20 & $31,5 \pm 2,12$ & $28-33$ & $>27$ \\
\hline
\end{tabular}

Legenda: Sem CA - sem compulsão alimentar; CA moderada - compulsão alimentar moderada; CA grave compulsão alimentar grave.

Tabela 4. Caracterização antropométrica da amostra. Niterói-RJ, 2017.

\begin{tabular}{|c|c|c|c|}
\hline & Média \pm DP & Min - Máx & Referências \\
\hline Peso $(\mathrm{kg})$ & $82,50 \pm 24,67$ & $48,70-143,9$ & - \\
\hline Estatura (m) & $164,6 \pm 8,62$ & $151-182$ & - \\
\hline IMC - eutrófico & $22,33 \pm 1,52$ & $20,33-24,51$ & $18,5-24,9 \mathrm{~kg} / \mathrm{m}^{2}$ \\
\hline IMC - sobrepeso & $27,02 \pm 1,80$ & $25,75-29,23$ & $25,0-29,9 \mathrm{~kg} / \mathrm{m}^{2}$ \\
\hline IMC - obesidade & $35,97 \pm 5,34$ & $30,04-49,21$ & $>30,0 \mathrm{~kg} / \mathrm{m}^{2}$ \\
\hline $\begin{array}{l}\text { Circunferência } \\
\text { abdominal }(\mathrm{H})\end{array}$ & $106,5 \pm 19,23$ & $79-142$ & $102 \mathrm{~cm}$ \\
\hline $\begin{array}{l}\text { Circunferência } \\
\text { abdominal (M) }\end{array}$ & $97,52 \pm 13,21$ & $78-124$ & $88 \mathrm{~cm}$ \\
\hline $\begin{array}{l}\text { Circunferência de } \\
\text { pescoço }(\mathrm{H})\end{array}$ & $43,5 \pm 2,12$ & $34-53,5$ & $37 \mathrm{~cm}$ \\
\hline $\begin{array}{l}\text { Circunferência de } \\
\text { pescoço }(M)\end{array}$ & $36,18 \pm 2,82$ & $31-45$ & $34 \mathrm{~cm}$ \\
\hline $\begin{array}{l}\text { Índice de } \\
\text { conicidade }(\mathrm{H})\end{array}$ & $1,29 \pm 0,08$ & $1,18-1,42$ & 1,28 \\
\hline $\begin{array}{l}\text { Índice de } \\
\text { conicidade (M) }\end{array}$ & $1,32 \pm 0,07$ & $1,16-1,45$ & 1,32 \\
\hline
\end{tabular}

Legenda: IMC - índice de massa corporal 
Tabela 5. Associação entre Transtorno da Compulsão Alimentar Periódica e observância à dieta, índice de massa corporal, circunferência abdominal, circunferência de pescoço e índice de conicidade. Niterói-RJ, 2017.

\begin{tabular}{|c|c|c|c|c|c|c|}
\hline $\begin{array}{c}\text { Observância à } \\
\text { dieta }\end{array}$ & $\mathrm{N}$ & $\%$ & $\begin{array}{l}\text { Sem Critérios } \\
\text { para TCAP }\end{array}$ & $\begin{array}{l}\text { Com critérios } \\
\text { para TCAP }\end{array}$ & $\begin{array}{l}\text { Valor } \\
\text { de P }\end{array}$ & $\mathrm{RC}$ \\
\hline $\begin{array}{l}\text { Observantes à } \\
\text { dieta } \\
\text { Não observantes } \\
\text { à dieta }\end{array}$ & 17 & 43 & 11 & 10 & $<0,05$ & 1,6 \\
\hline IMC & $\mathrm{N}$ & $\%$ & $\begin{array}{l}\text { Sem Critérios } \\
\text { para TCAP }\end{array}$ & $\begin{array}{l}\text { Com critérios } \\
\text { para TCAP }\end{array}$ & $\begin{array}{l}\text { Valor } \\
\text { de P }\end{array}$ & $\mathrm{RC}$ \\
\hline $\begin{array}{l}\text { IMC - eutrófico } \\
\text { IMC - sobrepeso } \\
\text { IMC - obesidade }\end{array}$ & $\begin{array}{c}11 \\
3 \\
16\end{array}$ & $\begin{array}{l}37 \\
10 \\
53\end{array}$ & $\begin{array}{l}9 \\
3 \\
7\end{array}$ & $\begin{array}{l}2 \\
0 \\
9\end{array}$ & $\begin{array}{c}- \\
- \\
<0,05\end{array}$ & $\begin{array}{c}- \\
- \\
1,4\end{array}$ \\
\hline CA & $\mathrm{N}$ & $\%$ & $\begin{array}{l}\text { Sem Critérios } \\
\text { para TCAP }\end{array}$ & $\begin{array}{c}\text { Com critérios } \\
\text { para TCAP }\end{array}$ & $\begin{array}{l}\text { Valor } \\
\text { de P }\end{array}$ & $\mathrm{RC}$ \\
\hline $\begin{array}{l}\text { Androide } \\
\text { Ginecoide }\end{array}$ & $\begin{array}{c}3 \\
27\end{array}$ & $\begin{array}{l}10 \\
90\end{array}$ & $\begin{array}{c}3 \\
16\end{array}$ & $\begin{array}{c}- \\
11\end{array}$ & 0,28 & 1,5 \\
\hline CP & $\mathrm{N}$ & $\%$ & $\begin{array}{c}\text { Sem Critérios } \\
\text { para TCAP }\end{array}$ & $\begin{array}{l}\text { Com critérios } \\
\text { para TCAP }\end{array}$ & $\begin{array}{l}\text { Valor } \\
\text { de P }\end{array}$ & $\mathrm{RC}$ \\
\hline $\begin{array}{l}\text { Adequado } \\
\text { Inadequado }\end{array}$ & $\begin{array}{l}11 \\
19\end{array}$ & $\begin{array}{l}37 \\
63\end{array}$ & $\begin{array}{c}8 \\
11\end{array}$ & $\begin{array}{l}3 \\
8\end{array}$ & 0,47 & 1,9 \\
\hline Índice $\mathrm{C}(\mathrm{H})$ & $\mathrm{N}$ & $\%$ & $\begin{array}{l}\text { Sem Critérios } \\
\text { para TCAP }\end{array}$ & $\begin{array}{l}\text { Com critérios } \\
\text { para TCAP }\end{array}$ & $\begin{array}{l}\text { Valor } \\
\text { de P }\end{array}$ & $\mathrm{RC}$ \\
\hline $\begin{array}{l}\text { Adequado } \\
\text { Inadequado }\end{array}$ & $\begin{array}{l}5 \\
5\end{array}$ & $\begin{array}{l}50 \\
50\end{array}$ & $\begin{array}{l}5 \\
2\end{array}$ & $\begin{array}{l}- \\
3\end{array}$ & 0,17 & 15,49 \\
\hline Índice C (M) & $\mathrm{N}$ & $\%$ & $\begin{array}{l}\text { Sem Critérios } \\
\text { para TCAP }\end{array}$ & $\begin{array}{l}\text { Com critérios } \\
\text { para TCAP }\end{array}$ & $\begin{array}{l}\text { Valor } \\
\text { de P }\end{array}$ & $\mathrm{RC}$ \\
\hline $\begin{array}{l}\text { Adequado } \\
\text { Inadequado }\end{array}$ & $\begin{array}{l}10 \\
10\end{array}$ & $\begin{array}{l}50 \\
50\end{array}$ & $\begin{array}{l}6 \\
6\end{array}$ & $\begin{array}{l}4 \\
4\end{array}$ & 1,00 & 1,00 \\
\hline
\end{tabular}

Legenda: sem critérios para TCAP - sem critérios sugestivos para transtorno da compulsão alimentar periódica (pacientes que apresentaram valores de Escala de Compulsão Alimentar < 17); com critérios para TCAP - com critérios sugestivos para transtorno da compulsão alimentar periódica (pacientes que apresentaram valores da Escala de Compulsão Alimentar > 18). IMC - índice de massa corporal 


\section{Discussão}

A população analisada no estudo, em sua maioria (63\%), foi classificada com sobrepeso e obesidade segundo os valores de IMC $\left(>25,0 \mathrm{~kg} / \mathrm{m}^{2}\right)$ e CP $(\mathrm{H}=43,5 \mathrm{~cm} \pm 2,12$ e M =36,18 cm $\pm 2,82)$ apresentados, além de possuírem valores médios de $\mathrm{CA}(\mathrm{H}=106,5 \mathrm{~cm} \pm 19,23$ e $\mathrm{M}=$ $97,52 \mathrm{~cm} \pm 13,21)$ e índice $\mathrm{C}(\mathrm{H}=1,29 \pm 0,08$ e $\mathrm{M}=1,32 \pm 0,07)$ maiores que os pontos de corte estabelecidos para a avaliação da obesidade abdominal.

Em seu estudo, Prisco et al. ${ }^{20}$ encontraram prevalência de TCAP similar entre homens e mulheres. Os mais jovens (até 35 anos de idade) apresentaram prevalência maior do que indivíduos acima de 35 anos. Apresentaram prevalências discretamente maiores os que tinham ensino fundamental/analfabeto.

No presente estudo, achados similares foram encontrados com relação aos sexos. A presença de critérios sugestivos foi de 30\% (n=3) para homens e $40 \%(\mathrm{n}=8)$ para mulheres, porém foi diferente com relação à idade $(64 \%, \mathrm{n}=7)$ dos pacientes que indicaram critérios apresentaram idades acima de 35 anos, e com relação à escolaridade $(55 \%, \mathrm{n}=6)$ dos pacientes que apresentaram critérios tinham ensino superior completo.

De acordo com o questionário ECAP, $37 \%(n=11)$ dos pacientes apresentaram critérios sugestivos para TCAP, percentual discretamente maior que os achados de Wietzikoski et al., ${ }^{21}$ em cujos estudos $20 \%$ da amostra foram classificadas com TCAP segundo os valores dos escores do ECAP.

Observou-se, no presente estudo, que pacientes que apresentaram critérios sugestivos para TCAP apresentaram pouca observância à dieta. De acordo com Spitzer et al., ${ }^{22}$ a maioria dos indivíduos com TCAP têm uma longa história de repetidas tentativas de fazer dietas e sentem-se desesperados com sua dificuldade de controlar a ingestão de alimentos. Alguns continuam tentando restringir o consumo de calorias, enquanto outros abandonam quaisquer esforços de fazer dieta, em razão de fracassos repetidos.

Masheb et al., ${ }^{23} \mathrm{em}$ seu estudo, examinaram as diferenças nos resultados da dieta, em que os pacientes que completaram o tratamento tiveram reduções significativas no IMC e na frequência de compulsão alimentar, bem como melhorias no aspecto psicológico. Evidenciaram, assim, benefícios da observância à dieta em pacientes com TCAP, demonstrando os benefícios tanto do diagnóstico como do tratamento de TCAP.

Wietzikoski et al. ${ }^{21}$ observaram, em estudo com participantes do sexo feminino com estado nutricional classificados em eutrofia, sobrepeso e obesidade, que $80 \%$ da amostra não apresentaram compulsão alimentar periódica, 15\% apresentaram compulsão alimentar periódica moderada e $5 \%$ apresentaram compulsão alimentar periódica severa, com média de 32,83 pontos. Verificouse que os indivíduos considerados obesos obtiveram escores mais elevados quando comparados aos eutróficos, sugerindo que o IMC elevado pode estar associado ao desenvolvimento de TCAP. 
No presente trabalho, resultados similares foram encontrados, pois pacientes com critérios sugestivos para TCAP apresentaram altos valores de IMC, sendo classificados como obesos. Mostrou-se assim a importância de oferecer um tratamento adequado com vistas às limitações que esses pacientes possam apresentar durante o tratamento nutricional. Porém, a especificidade destes pacientes deve ser considerada para que o tratamento tenha sucesso, já que as compulsões são extremamente deletérias a qualquer tentativa de perda de peso. ${ }^{24,25}$

A circunferência abdominal (CA) avalia a adiposidade subcutânea e visceral. É facilmente mensurada e frequentemente usada como uma medida de gordura visceral em estudos epidemiológicos. ${ }^{26,27} \mathrm{Em}$ seu estudo, Mosca et al. ${ }^{28}$ analisaram a circunferência abdominal de grupos com TCAP e sem TCAP, tendo como pontos de referências $94 \mathrm{~cm}$ em homens e $80 \mathrm{~cm}$ para mulheres. Verificaram que homens sem TCAP apresentaram circunferência abdominal menores que homens com TCAP, e que mulheres sem TCAP apresentaram circunferência abdominal maiores que mulheres com TCAP. No presente estudo, não foi encontrada associação significativa com a presença de critérios para TCAP.

Silva et al. ${ }^{29}$ analisaram a circunferência de cintura de indivíduos adultos com obesidade, de ambos os sexos (22 com e 17 sem TCAP), usando como parâmetros circunferência de cintura $\geq 88$ $\mathrm{cm}$ na mulher e $102 \mathrm{~cm}$ no homem, e tendo como indicador o mesmo ponto de corte do presente estudo. A circunferência da cintura foi acima dos cortes recomendados para ambos os grupos. No presente estudo, achados similares foram encontrados: tanto homens como mulheres, com e sem critérios sugestivos para TCAP, apresentaram valores acima do ponto de corte, dificultando assim a análise estatística.

Segundo Tibana et al., ${ }^{30}$ a CP pode ser um parâmetro confiável para indicar riscos cardiovasculares, quando comparado à gordura depositada na região visceral. Os autores destacam que a medida da CP apresenta forte relação com IMC, hipertensão arterial e indicadores bioquímicos de resistência à insulina e riscos cardiometabólicos, podendo ser usada como boa ferramenta de identificação das perturbações precoces do metabolismo. No presente estudo, a população analisada apresentou valores de CP acima da normalidade, demonstrando alto risco para doenças cardiovasculares no público analisado, não caracterizando, entretanto, uma associação significativa com a presença ou não de critérios para TCAP.

Andrade et al. ${ }^{31}$ observaram o índice C em mulheres e sua associação com hipertensão e diabetes mellitus. Aquelas que apresentavam altos valores de índice C tiveram $72 \%$ e $75 \%$ mais chance de ter diabetes mellitus e hipertensão, respectivamente.

Pitanga \& Lessa ${ }^{32}$ sugeriram 1,18 como o melhor ponto de corte para o índice C, apresentando valores de 73,39\% para sensibilidade e de $61,15 \%$ para especificidade e área sob a curva ROC de 0,75 para mulheres. Para homens, recomendaram o índice C com ponto de corte de 1,23 apresentando 
valores de 73,91\% para sensibilidade e de 74,92\% especificidade e área sob a curva ROC de 0,80. Eles concluíram que o índice $\mathrm{C}$ pode ser utilizado para discriminar o risco cardiovascular, mesmo que a sensibilidade e a especificidade não sejam muito altas. No entanto, existe a possibilidade de classificações incorretas levando a um maior número de resultados falso-positivos.

Na presente pesquisa, foram utilizados 1,28 para homens e 1,32 para mulheres, como valores da mediana da amostra, como ponto de corte para o índice C, pois apenas $17 \%(n=5)$ da população apresentava índice $\mathrm{C}<1,18$ e 1,23. Este parâmetro não apresentou associação estatística significativa nos grupos de homens e mulheres $(\mathrm{p}=0,17$ e $\mathrm{p}=1,00)$ respectivamente.

Cabe enfatizar que este trabalho apresentou algumas limitações, como o número reduzido de pacientes em cada grupo e o fato de ser um estudo transversal. Entretanto, como foram identificados indivíduos obesos na população em estudo, é importante incentivar práticas que levem à perda de peso, pois reduzir o peso garante não só menor morbidade como significativa melhoria no estado emocional e comportamental. O tratamento nutricional, portanto, tem como principal objetivo reverter as alterações do estado nutricional provocadas pela compulsão alimentar e promover hábitos alimentares mais saudáveis, restabelecendo a saúde do indivíduo. ${ }^{33}$

\section{Conclusão}

Constatou-se que, na população analisada, existem pacientes com critérios sugestivos para TCAP. Foi observado que os pacientes com critérios apresentaram pouca observância a dieta, o que pode ser explicado pelos episódios de compulsão alimentar.

Quanto ao estado nutricional dos pacientes, observou-se associação entre IMC e critérios sugestivos para TCAP, pois pacientes que apresentaram critérios estavam acima do peso. Com relação a circunferência de abdominal, circunferência de pescoço e índice de conicidade, não foram encontradas associações significativas.

\section{Colaboradores}

Silva CA e Grassano KBC participaram das etapas de concepção do projeto, desenho, análise e interpretação dos resultados. Barroso SG e Rocha GS participaram de todas as etapas do processo, desde a concepção do projeto, orientação, correção, análise, interpretação dos resultados e aprovação final do artigo.

Conflito de Interesses: Os autores declaram não haver conflito de interesses. 


\section{Referências}

1. Flegal KM, Carrol MD, Kit BK, Ogden CL. Prevalence of obesity and trends in the distribution of body mass index among US adults, 1999-2010. JAMA 2012; 307(5):491-497.

2. Hilton S, Patterson C, Teyhan A. Escalating coverage of obesity in UK newspapers: the evolution and framing of the "Obesity Epidemic" from 1996 to 2010. Obesity 2012; 20(8):1688-1695.

3. Gigante DP, Moura EC, Sardinha LMV. Prevalência de excesso de peso e obesidade e fatores associados, Brasil, 2006. Rev Saúde Pública 2009; 43(Supl. 2):83-89.

4. American Diabetes Association. Standards of medical care in Diabetes 2014. Diabetes Care 2014; 37(Supl. 1):14-80.

5. World Health Organization. Global status report on communicable diseases 2014. Geneva: WHO; 2014.

6. Brasil. Ministério da Saúde. Secretaria de Atenção à Saúde. Departamento de Atenção Básica. Política Nacional de Alimentação e Nutrição. Brasília: Ministério da Saúde; 2012.

7. Wyatt HR. Update on treatment strategies for Obesity. J Clin Endocrinol Metab. 2013; 98(4):1299-1306.

8. Chaput JP, Klingenberg L, Astrup A, Sjödin AM. Modern sedentary activities promote overconsumption of food in our current obesogenic environment. Obes Rev. 2011; 12(5):e12-20.

9. American Psychiatric Association. Diagnostic and statistical manual of mental disorders DSM-V. 5th ed. Washington DC: APA; 2013.

10. Wonderlich SA, Gordon KH, Mitchell JE, Crosby RD, Engel SG. The validity and clinical utility of binge eating disorder. Int J Eat Disord. 2009; 42(8):687-705.

11. Nunes MA. Transtornos alimentares e obesidade. $2^{\circ}$ ed. Porto Alegre: Artmed; 2009.

12. Cubrelati BS, Rigoni PAG, Vieira LF, Belem IC. Relação entre distorção de imagem corporal e risco de desenvolvimento de transtornos alimentares em adolescentes. Revista da Faculdade de Educação Física da UNICAMP 2014; 12(1):1-15.

13. Freitas S, Lopes CS, Coutinho W, Appolinario JC. Tradução e adaptação para o português da Escala de Compulsão Alimentar Periódica. Rev Bras Psiquiatr. 2001; 23(4):215-220.

14. Petribu K, Ribeiro ES, Oliveira FMF, Braz CIA, Gomes MLM, Araujo DE, et al. Transtorno da compulsão alimentar periódica em uma população de obesos mórbidos candidatos a cirurgia bariátrica do Hospital Universitário Oswaldo Cruz, em Recife - PE. Arq Bras Endocrinol Metab. 2006; 50(5):901-908.

15. World Health Organization. Obesity: preventing and managing the global epidemic. Report of a World Health Organization consultation. Geneva: World Health Organization; 2000. 256 p. WHO obesity technical report series, 284.

16. Third Report of the National Cholesterol Education Program (NCEP) Expert Panel on Detection, Evaluation, and Treatment of High Blood Cholesterol in Adults (Adult Treatment Panel III) final report. Circulation 2000; 106(25):3143-3421. 
17. Alberti KG, Eckel RH, Grundy SM, Zimmet PZ, Cleeman JI, Donato KA, et al. Harmonizing the metabolic syndrome: a joint interim statement of the International Diabetes Federation Task Force on Epidemiology and Prevention; National Heart, Lung, and Blood Institute; American Heart Association; World Heart Federation; International Atherosclerosis Society; and International Association for the Study of Obesity. Circulation 2009; 120(16):1640-1645.

18. Ben-Noun L, Sohar E, Laor A. Neck circumference as a simple screening measure for identifying overweight and obese patients. Obes Res. 2001; 9(8):470-477.

19. Valdez R. A simple model-based index of abdominal adiposity. J Clin Epidemiol. 1991; 44(9):955-956.

20. Prisco APK, Araújo TM, Almeida MMG, Santos KOB. Prevalência de transtornos alimentares em trabalhadores urbanos de município do Nordeste do Brasil. Ciênc Saúde Coletiva 2013; 18(4):1109-1118.

21. Wietzikoski EC, Anelli D, Sato SW, Costa LD, França VF. Prevalência de compulsão alimentar periódica em indivíduos do sudoeste do Paraná. Arq Cienc Saúde UNIPAR 2014; 18(3):173-179.

22. Spitzer RL, Devlin M, Walsh BT, Hasin D, Wing R, Marcus M, et al. Binge eating disorder: a multisite field trial of the diagnostic criteria. Int J Eat Dis. 1992; 11(3):191-203.

23. Masheb RB, Dorflinger LM, Rolls BJ, Mitchell DC, Grilo CM. Binge abstinence is associated with reduced energy intake after treatment in patients with binge eating disorder and obesity. Obesity 2016; 24(12):2491-2496.

24. Becker AE, Grinspoon SK, Klibanski A, Heizog DB. Eating disorders. N Engl J Med. 1999; 40(14):1092-1098.

25. Borges MBF, Jorge MR. Evolução histórica do conceito de Compulsão Alimentar. Psiq Prat Med. 2000; 33(4):113-118.

26. Folsom AR, Kushi LH, Anderson KE, Mink PJ, Olson JE, Hong CP, et al. Associations of general and abdominal obesity with multiple health outcomes in older women: the Iowa Women's Health Study. Arch Intern Med. 2000; 160(14):2117-2128.

27. Rexrode KM, Carey VJ, Hennekens CH, Walters EE, Colditz GA, Stampfer MJ, et al. Abdominal adiposity and coronary heart disease in women. JAMA1998; 280(21):1843-1848.

28. Mosca LN, Costa LRLG, Ramos CFC, Asano LMT, Ferreira AD. Compulsão alimentar periódica de pacientes em tratamento para redução de peso. J Health Sci Inst. 2010; 28(1):59-63.

29. Silva HGV, Magalhães VC, Oliveira BA, Rosa JS, Santos TT, Moreira AB. Características antropométricas e metabólicas em obesos com transtorno alimentar. Revista HUPE 2015; 14(3):14-19.

30. Tibana RA, Teixeira TG, Farias DL, Silva AO, Madrid B, Vieira A, et al. Relação da circunferência do pescoço com a força muscular relativa e os fatores de risco cardiovascular em mulheres sedentárias. Einstein 2012; 10(3):329-334.

31. Andrade MD, Freitas MC, Sakumoto AM, Pappiani C, Andrade SC, Vieira VL, et al . Association of the conicity index with diabetes and hypertension in Brazilian women. Arch Endocrinol Metab. 2016; 60(5):436-442. 
32. Pitanga FJG, Lessa I. Indicadores antropométricos de Obesidade como instrumento de triagem para risco coronariano elevado em adultos na cidade de Salvador - Bahia. Arquivos Brasileiros de Cardiologia 2005; 85(1):26-31.

33. Capuron L, Poitou C, Machaux-Tholliez D, Frochot V, Bouillot JL, Basdevant A, et al. Relationship between adiposity, emotional status and eating behaviour in obese women: role of inflammation. Psychol Med. 2011; 41(7):1517-1528.

Recebido: 28 de junho, 2017

Revisado: 14 de outubro, 2017

Aceito: 24 de outubro, 2017 